\title{
Design Optimization of a Multi-kW Thermoacoustic Electric Generator Using DeltaEC Model
}

\author{
Srinath Somu ${ }^{1}$, Deanna Lacoste ${ }^{1}$, Saumitra Saxena ${ }^{1}$, William L. Roberts ${ }^{1}$, Robert M. Keolian ${ }^{2}$ \\ ${ }^{1}$ Clean Combustion Research Center, \\ King Abdullah University of Science and Technology, Thuwal, Saudi Arabia. \\ ${ }^{2}$ Sonic Joule LLC, State College, Pennsylvania, USA.
}

\begin{abstract}
Waste heat recovery from power plants and industries requires a new type of electricity generators and related technological developments. The current research work is aimed at the design of a multi-kilowatt thermoacoustic electric generator, which can be employed as the bottoming cycle of a gas-turbine power plant or for industrial waste heat recovery. The proposed device converts thermal energy into acoustic power and subsequently uses a piezoelectric alternator to convert acoustic power into electricity. The challenge in designing such a device is that it has to be acoustically balanced and the performance of the device is greatly affected by numerous parameters such as frequency of the traveling acoustic wave, heat exchanger parameters, regenerator dimensions, acoustic feedback loop, etc. The proposed device is a lab-scale demonstration targeted to produce a few kilowatts of electric power from a $20 \mathrm{~kW}$ th heat source. To achieve the acoustically balanced configuration of the device, DeltaEC software is used. The DeltaEC model outcomes are used to arrive at the optimized design of the device and its components. The analytical method, the optimized geometrical dimensions of thermoacoustic components and the minimum required conditions of heat source input are presented in this paper.
\end{abstract}

Keywords: thermoacoustic, piezoelectric alternator, waste heat, acoustic, DeltaEC, energy conversion, bottoming cycle, gas-turbine

\section{NOMENCLATURE}

English letters

a

sound speed, $\mathrm{m} / \mathrm{s}$
A area, $\mathrm{m}^{2}$

$c_{p} \quad$ specific heat at constant pressure, $\mathrm{J} / \mathrm{kg} \cdot \mathrm{K}$,

$f \quad$ friction factor

$\dot{H} \quad$ rate at which total energy flows (total power), $\mathrm{W}$

$k \quad$ thermal conductivity, $\mathrm{W} / \mathrm{m} . \mathrm{K}$

$\dot{M} \quad$ mass flow, $\mathrm{kg} / \mathrm{s}$

$p \quad$ pressure, $\mathrm{N} / \mathrm{m}^{2}$

$r$ resistance

$R \quad$ gas constant, $\mathrm{J} / \mathrm{kg} \cdot \mathrm{K}$

$T \quad$ temperature, $\mathrm{K}$

$u \quad \mathrm{x}$ component of velocity, $\mathrm{m} / \mathrm{s}$

$U$ volume flow rate, $\mathrm{m}^{3} / \mathrm{s}$

\section{Greek Letters}

$\rho$ density, $\mathrm{kg} / \mathrm{m}^{3}$

$\mu \quad$ dynamic viscosity, $\mathrm{kg} / \mathrm{m} . \mathrm{s}$

$\omega \quad=2 \pi f ;$ angular frequency, $\mathrm{s}^{-1}$,

$\gamma \quad$ ratio of isobaric to isochoric specific heats

\section{Subscripts}

0 'environment' or 'ambient'

1 first order, usually complex amplitude

2 second order

$\mathrm{m}$ mean

$\kappa \quad$ thermal diffusivity

$v \quad$ kinematic viscosity

\section{Special Notations}

$\operatorname{Re}[$ ] Real part of a complex term

$\operatorname{Im}[$ ] Imaginary part of a complex term 


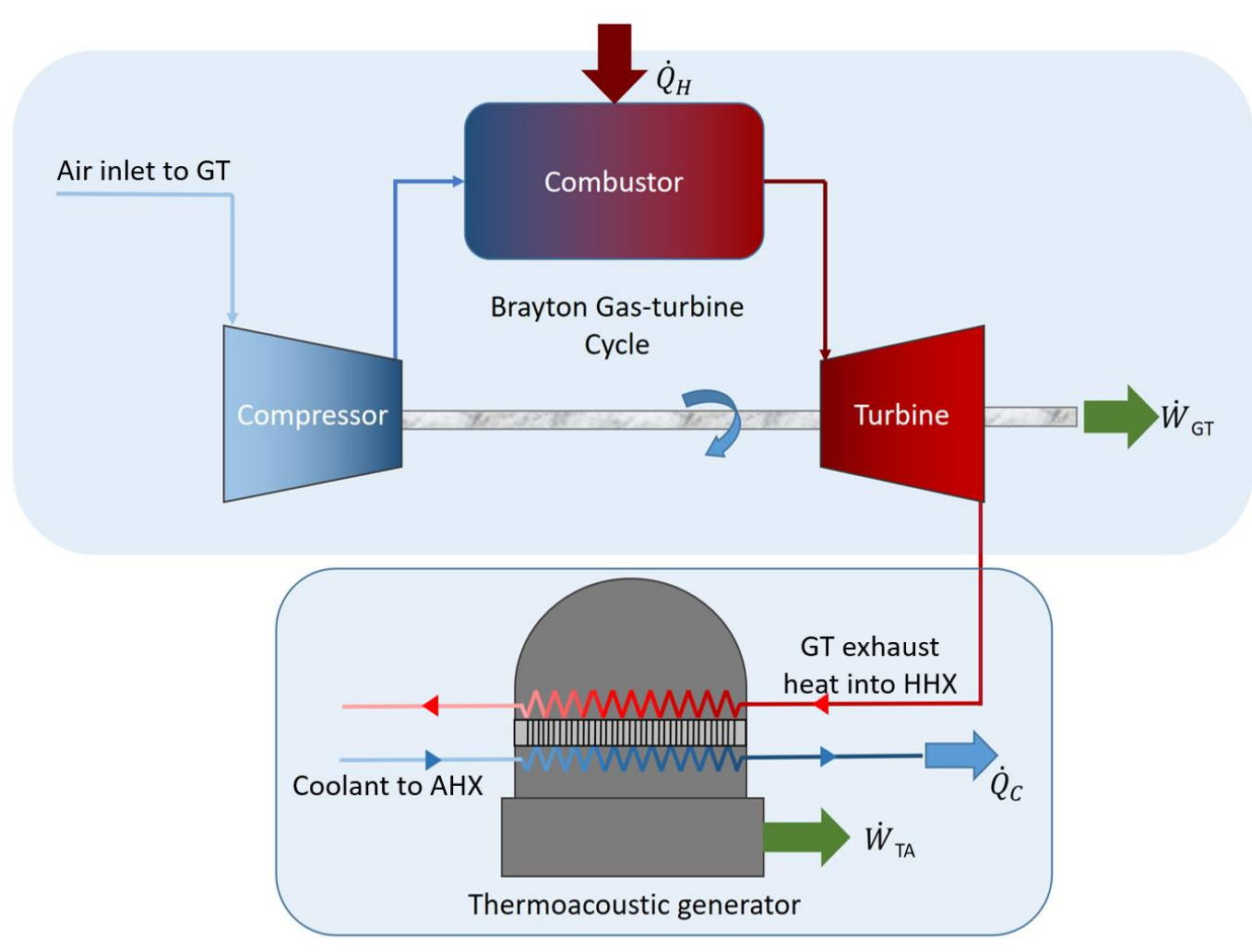

\section{Figure 1 SCHEMATIC REPRESENTATION OF THERMOACOUSTIC ELECTRIC GENERATOR COMBINED WITH A SIMPLE GAS-TURBINE CYCLE}

\section{INTRODUCTION}

Valorizing the low-to-medium quality $\left(300-600^{\circ} \mathrm{C}\right)[1]$ waste heat from power generation and industrial sectors with low cost $(\sim \$ 1000 / \mathrm{kW})$ and high efficiency $(\sim 30-40 \%$ of Carnot per kilowatt) is imperative for better utilization of dwindling fossil resources and carbon footprint reduction. Even though the existing bottoming cycle such as the steam Rankine cycle, organic Rankine cycle or Kalina cycle have proven merits in terms of its efficiencies, the cycle consumes a significant amount of energy as latent heat for the continuous operation. Moreover, these bottoming cycles are mechanically too complex and occupy a lot of space [2]. Therefore these bottoming cycles are not economically viable options for combined cycle power plants. As an alternate option, recent advancements have shown that thermoacoustic technology has a significant potential for converting waste heat to power [3]. The thermoacoustic device can be cost-effective when compared to the above mentioned bottoming cycle power plants due to that the working fluid does not change its phase except generating acoustical oscillations; it has a compact design and almost no moving parts. A simple illustration of how a thermoacoustic device can be coupled with a simple-cycle gas turbine as a bottoming cycle is shown in Figure 1.

Figure 1 represents that a simple-gas turbine power plant converts thermal energy $\dot{Q}_{H}$ into the electrical power output $\dot{W}_{G T}$. The hot exhaust gas from the turbine is typically at a temperature range of $300^{\circ} \mathrm{C}$ to $500^{\circ} \mathrm{C}$. This waste heat is the potential source for the thermoacoustic electric generator. The device consists of a hot heat exchanger (HHX) and an ambient heat exchanger (AHX) and in between the hot heat exchanger and ambient heat exchanger, a porous material with a certain thickness is provided, which is called a regenerator. This arrangement of a hot heat exchanger, regenerator, and ambient heat exchanger is called 'thermal-core', which is enclosed in a pressure vessel.

In thermoacoustic engines, the working fluid, generally a noble gas, is caused to interact with a regenerator, which is maintained at a high-temperature gradient. At the regenerator, the working fluid experiences thermal expansion when the pressure is high and thermal contraction when the pressure is low [4]. Thus the thermal interaction between the gas and surface of the porous material generates self-sustained acoustical oscillations, which convert heat into mechanical energy in the form of sound. The sound can either be predominantly in a standing wave or traveling wave depending on the size of the pores in the regenerator and the acoustic environment created by a resonator enclosing the sound. This acoustic power is converted into electrical power $\dot{W}_{T A}$ by using a suitable alternator, which will be explained later.

In order to estimate the potential of waste heat valorization, GE Company's LM6000-PF+ gas turbine is taken as an example for the calculation [5]. The rated power output of this gas turbine 
is $52 \mathrm{MW}$; heat rate LHV (Lower heating value) is $8656 \mathrm{~kJ} / \mathrm{kW}$ $\mathrm{hr}$ and the LHV based efficiency is $41.6 \%$. The exhaust flow rate is $136 \mathrm{~kg} / \mathrm{s}$ at a temperature of $501^{\circ} \mathrm{C}$. When a thermoacoustic electric generator is combined with this gas turbine exhaust the additional power generated from the waste heat is expected to be significant. When using the waste heat from the combustion products, the sizeable drop in the hot temperature must be considered as heat is pulled out of the gas and results in a change in Carnot and thermal efficiencies. If the Carnot efficiency of the thermoacoustic device is assumed to be $35 \%$ without considering the temperature drop, then all its LHV 'heat' is extracted. Then the absolute maximum amount of acoustic work that can be extracted is 9.8 MW. In actuality, the Carnot efficiency decreases as the hot temperature approaches the ambient. So, at some point, the extraction of heat out of hot gas should be stopped as being uneconomical. Also, the output power can be calculated by an analytical integration over the temperature drop, assuming a certain fraction of Carnot efficiency held constant as the hot gas temperature drops. The penalty of not extracting the full LHV of the gas and the power output at steps of temperature drop is shown in Figure 2.

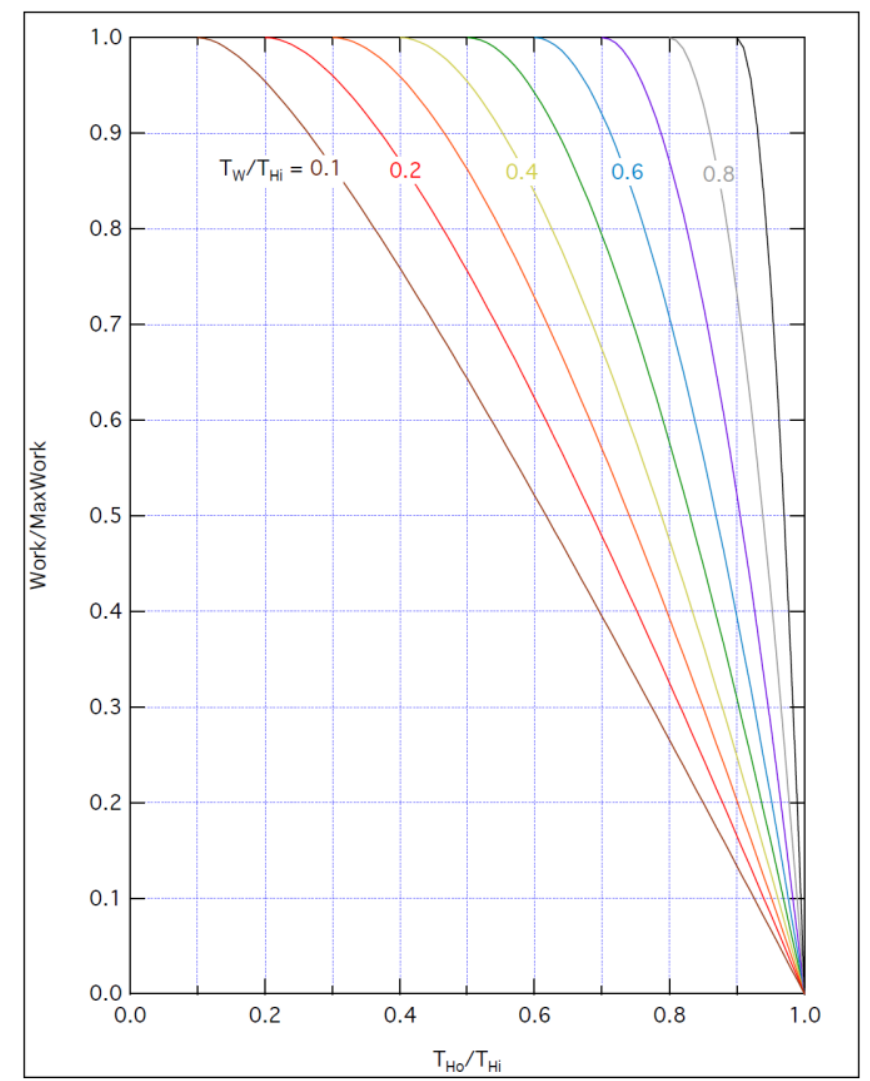

Figure 2 VALORISATION OF WASTE HEAT FROM GE LM6000PF GAS-TURBINE

The vertical axis shows the amount of work extracted, in other words, the sound generated in the device, scaled by the maximum work of $9.8 \mathrm{MW}$, assuming the approximations of
$35 \%$ of Carnot throughout, a constant combustion product heat capacity (chosen at a representative middle hot temperature), and a constant cooling-water cold-side temperature of $T_{w}$.

In other words, the vertical axis can be pretty much read as the power extraction going from 0 to $10 \mathrm{MW}$. The horizontal axis is the ratio of the hot outlet temperature $T_{\mathrm{Ho}}$ to the hot inlet temperature $T_{\mathrm{Hi}}$. The LM6000-PF+ outlet temperature is $774 \mathrm{~K}$, and for a cooling water temperature of $318 \mathrm{~K}\left(45^{\circ} \mathrm{C}\right)$ the ratio of $T_{\mathrm{W}}$ to $T_{\mathrm{Hi}}$ is 0.41 . In Figure 2 the yellowish line for $T_{\mathrm{W}} / T_{\mathrm{Hi}}=0.40$ (right on the yellow line corresponds to $T_{\mathrm{W}}=37^{\circ} \mathrm{C}$ ). The graph is saying that about $4.7 \mathrm{MW}$ of sound could be generated as $T_{\mathrm{Ho}} / T_{\mathrm{Hi}}$ cools the first third, from 1.0 to 0.8 ; another $3.6 \mathrm{MW}$ could be generated as $T_{\mathrm{Ho}} / T_{\mathrm{Hi}}$ cools the second third, from 0.8 to 0.6 ; and 1.7 MW could be generated if we bothered to cool the combustion products $\mathrm{T}_{\mathrm{Ho}} / \mathrm{T}_{\mathrm{Hi}}$ over its last third from 0.6 to 0.4. In other words, it is possible to get about $83 \%$ of the available $10 \mathrm{MW}$, about $8.3 \mathrm{MW}$, if the waste heat from LM6000-PF+ combustion products is cooled two-thirds of the way to $\mathrm{T}_{\mathrm{W}}$, from $774 \mathrm{~K}\left(501^{\circ} \mathrm{C}\right)$ to $470 \mathrm{~K}\left(197^{\circ} \mathrm{C}\right)$. This extraction of power from waste heat is a motivating factor in the field of power generation.

The early thermoacoustic generators developed by Bell Telephone Laboratories in 1951 [6] and in 1958 [7] on singing pipes produced standing waves and generated electrical power by coupling to acoustical-to-electrical transducers. Later it was found that standing wave engines, with pressure and velocity swings of the working fluid nearly $90^{\circ}$ out of phase, tend to be inefficient. They generally rely on a regenerator with relatively large pores and relatively poor thermal contact between the working fluid and regenerator. This produces a thermal lag between working fluid compression and heat transfer to the regenerator to get the necessary phasing between thermal expansion and pressure swings in the working fluid that provides amplification of the sound. The poor thermal contact, however, leads to entropy production that lowers the efficiency of the engine. In contrast, in traveling wave engines, with pressure and velocity swings of the working fluid nearly in phase, the sound itself provides the necessary phasing between thermal expansion and heat transfer to the regenerator with relatively small pores. When the pores are small the heat transfer is nearly isothermal, entropy production is low, and engine efficiency is nearly doubled. Thus, our acoustic resonator is in the form of a loop, with a traveling wave of sound propagating clockwise as shown in FIGURE 3, in an engine style first envisioned by Ceperley [8]. The traveling wave heat engine might be considered similar to Stirling engines which use a column of fluid as the piston [9].

Researchers around the world have attempted to develop thermoacoustic electric generators based on the following techniques for acoustic to electrical conversion:

(i) Electro-magnetic devices (linear alternator),

(ii) Magneto-hydrodynamic devices,

(iii) Bi-directional turbines, and

(iv) Piezoelectric alternators. 


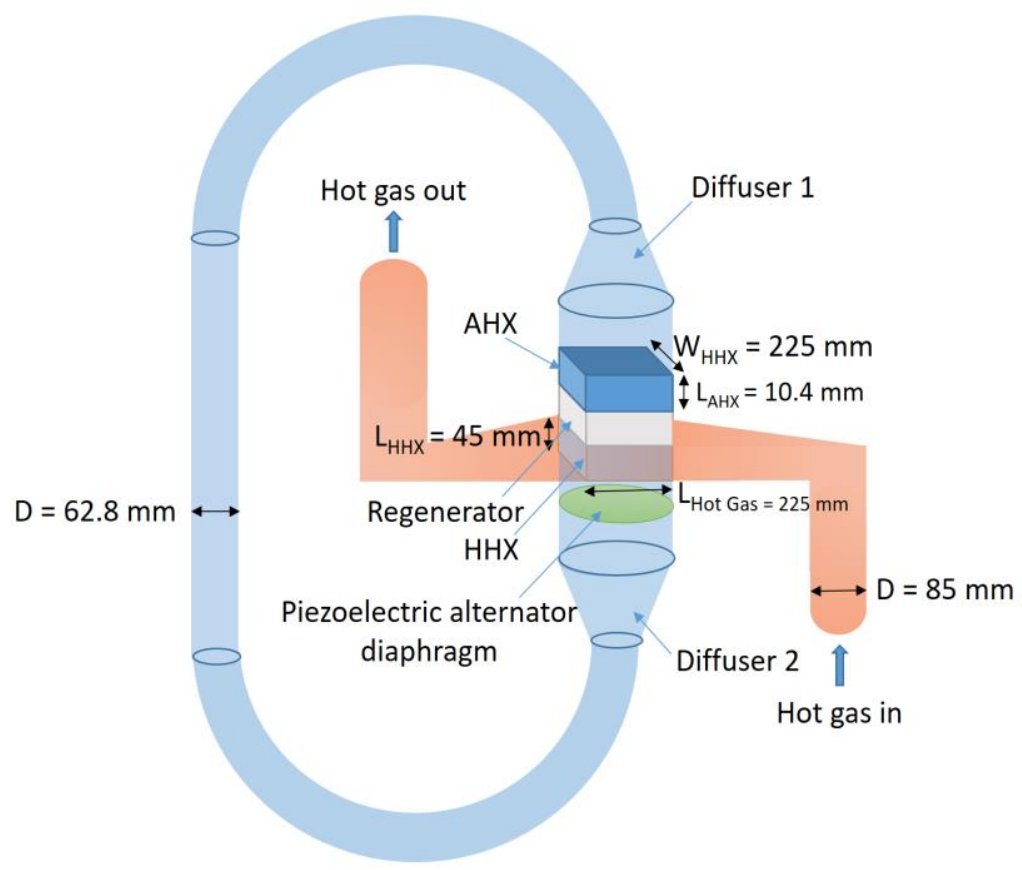

FIGURE 3. SCHEMATIC OF SINGLE THERMAL-CORE THERMOACOUSTIC ELECTRIC GENERATOR (ST-TEG).

In the case of the first technique, the linear alternator is found to be suitable for producing electricity in kilowatt range from the acoustic power [10] [11]. But scaling the linear alternator for much larger power output would be highly challenging [3] due to the large amplitude oscillation of the vibrating piston and difficulty in maintaining the minimum clearance between the piston and its cylinder. The second technique, i.e. the application of magneto-hydrodynamic transducers for the conversion of acoustic to electricity was successfully implemented by Swift [12], using liquid sodium at 200 bar as the working fluid. It was shown that the engine performed well at an operating frequency of $1000 \mathrm{~Hz}$. But at high pressure the entire engine can cause problems in sealing the electrodes and generating the acoustic oscillations [13].

The next technique, the application of a bi-directional turbine in thermoacoustic conversion is relatively new and shows a competing performance with other techniques at low power output. It is proposed that combining the oscillating water column with the thermoacoustic device would make this technique feasible for large scale power generation [14]. But, the bi-directional turbine technique needs additional investigation.

Based on the piezoelectric alternator technique, Keolian et al. demonstrated an electrical power output of $37 \mathrm{~W}$ from a thermoacoustic engine as a proof of concept in preparation for the valorization of the exhaust heat from a Volvo truck engine [15]. Among these techniques, we believe that a flexible diaphragm piezoelectric alternator can be viable to produce higher electrical power output and that is more easily scaled-up. In this type of thermoacoustic generator, the piezoelectric alternator is aligned inline with a thermoacoustic engine thermal- core, which is the combined configuration of a hot heat exchanger, regenerator, and an ambient heat exchanger. The schematic of the single thermal-core thermoacoustic electric generator (ST-TEG) is shown in FIGURE 3.

The acoustic oscillations generated in the thermal-core resonate along with a thin metal diaphragm of the alternator. The schematic representation of the piezoelectric alternator is shown in Figure 4. The diaphragm is supported by a ring of piezoelectric stacks interconnected by mechanical mounts. As the diaphragm flexes in either direction, it pulls inward on the piezoelectric stacks causing a large amplified fluctuating compressive stress in the elements, which then convert the stress into electricity with high efficiency [16]. The diaphragm is made up of high carbon steel. The operating frequency is about $300 \mathrm{~Hz}$.

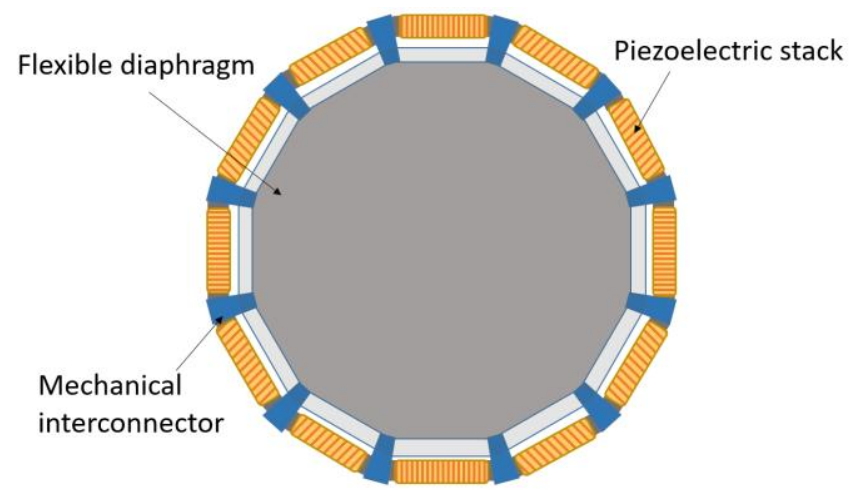

Figure 4. SCHEMATIC OF PIEZOELECTRIC ALTERNATOR 
The fatigue curves of high carbon steel ensure that the diaphragm has a near-infinite lifetime [17] if stress is kept below the fatigue endurance limit, which is needed because of the relatively high operating frequency. Therefore, the thermoacoustic device can be free of internal wearing parts.

The present research work is aimed at the design and development of a single thermal-core thermoacoustic device to generate multi-kilowatts of electricity using a piezoelectric alternator. For this device, it has been decided to supply hot gas at a temperature of around $500^{\circ} \mathrm{C}$ sourced from a $20 \mathrm{~kW}_{\text {th }}$ burner. Helium gas at 20 bar is the working fluid of the device.

The hot gas from the burner is supplied to a rectangular hot heat exchanger (HHX) as shown in FIGURE 3. Above the HHX, a stack of fine-mesh stainless steel screen is positioned, which acts as the regenerator, over which ambient heat (AHX) is aligned inline. The thermal-core configuration is enclosed in a pressure chamber, in which the piezoelectric alternator diaphragm is mounted below the HHX. The chamber is connected with a feedback tube through diffusers on both ends, to allow the acoustic wave to go around the loop. The most challenging aspect in the design of such a thermoacoustic device is optimizing the efficiency and power output of the generator, which depends on the dimensions of many components, such as the thicknesses (lengths in the vertical acoustical direction) of the hot heat exchanger $L_{\mathrm{HHX}}$, regenerator $L_{\mathrm{Reg}}$, and ambient heat exchanger $L_{\mathrm{AHX}}$; cross-sectional areas of thermal-core, diffuser inlet, and outlets; length and cross-sectional area of the feedback tubes; the dimensions of porous tubes of the heat exchangers and also hot exhaust gas path configuration in the hot heat exchanger. In addition, the materials of the components play a major role in the heat and mass transfer across the device. Therefore it is necessary to do thermal, flow, structural and acoustical analysis for designing a thermoacoustic device. DeltaEC software has been used to carry out these analyses. This paper explains the methodology adopted for obtaining the design of the single thermal core thermoacoustic engine using DeltaEC software.

\section{DeltaEC Simulation}

DeltaEC (Design Environment for Low-amplitude Thermoacoustic Energy Conversion) is a software developed by Los Alamos National Laboratory [18] [19], in which a thermoacoustic engine or other acoustical systems can be simulated for its performance evaluation. DeltaEC describes a device as a one-dimensional series of segments, which it evaluates in the low-amplitude acoustic approximation. It integrates the real and imaginary components of the acoustic pressure $p_{1}$, the real and imaginary parts of the acoustic volumetric velocity $U_{1}$, and the mean temperature $T_{m}$ in one dimension along the segments from one end of the device to the other. It does so analytically where it can and numerically where it must, so that operation is fast enough that many iterations can be accomplished quickly. It is not a CFD program.

A DeltaEC model can have many natural inputs, such as the dimensions of parts described by the segments, values at the start of the segment list for $p_{1}, U_{1}$ and $T_{m}$, and energy inputs such as the heats into heat exchangers or power into transducers. And the model has many natural outputs, such as $p_{1}, U_{1}, T_{m}$, and energy flows throughout the device and predictions of temperature drops in the heat exchangers. A key feature of the DeltaEC program is its ability to iteratively "guess" a number of natural inputs to "target" an equal number of user-desired values for the natural outputs, in effect swapping natural inputs to become outputs while simultaneously swapping natural outputs to become inputs. For example, in our engine, which is an annular loop, we allow four of the dimensions of the engine to be guessed so that the four values of the complex components of $p_{1}$ and $U_{1}$ at the end of the model are identical to the values at the beginning of the model, thus enforcing periodic boundary conditions. The following differential equations in the wave propagation direction $x$ are integrated in DeltaEC from its initial segment to the end of the final segment.

The $x$ component of the momentum equation is given by

$$
\frac{d p_{1}}{d x}=-i \omega \rho_{m} u_{1}+\mu\left[\frac{\partial^{2} u_{1}}{\partial y^{2}}+\frac{\partial^{2} u_{1}}{\partial z^{2}}\right] \text {. }
$$

The $x$ component of the continuity equation is given by

$$
\frac{d u_{1}}{d x}=-i \omega \frac{p_{1}}{\rho_{m} a^{2}}
$$

where $a=\sqrt{\gamma R_{u} T / M}, R_{u}$ is the universal gas constant and $M$ is the molecular weight of the working fluid.

The $x$ component of energy equation or the general thermoacoustic equation is given by

$$
\begin{aligned}
& {\left[1+(\gamma-1) f_{k}\right] p_{1}+\frac{\gamma p_{m}}{\omega^{2}} \frac{d}{d x}\left(\frac{1-f_{v}}{\rho_{m}} \frac{d p_{1}}{d x}\right)-} \\
& \frac{a^{2}}{\omega^{2}} \frac{f_{k}-f_{v}}{1-\sigma} \frac{1}{T_{m}} \frac{d T_{m}}{d x} \frac{d p_{1}}{d x}=0,
\end{aligned}
$$

where $f_{k}$ and $f_{v}$ are the so-called thermal and viscous Rott functions that take into account the thermal and viscous interactions of the sound with solid walls, and $\sigma$ is the Prandtl number.

The total power $\dot{H}_{t o t}$ is generally independent of $x$ except in the regions of heat exchangers, electroacoustic transduces, and at begin and end conditions. The total power including the steady flow streaming condition is given by

$$
\begin{aligned}
& \dot{H}_{\text {tot }}=\dot{M}_{2} c_{p}\left(T_{m}-T_{o}\right)+\frac{1}{2} \rho_{m} c_{p} \int \operatorname{Re}\left[T_{1} \widetilde{u_{1}}\right] d A- \\
& \left(A k-A_{\text {solid }} k_{\text {solid }}\right) \frac{d T_{m}}{d x} .
\end{aligned}
$$

The $x$ component for the static component of second-order mass flow (indicated by the 2.0 subscript) called Gedeon Streaming, is given by

$$
-\frac{d p_{2,0}}{d x}+r_{v, 2,0} U_{2,0} \simeq 0
$$

In addition to equations (1) to (5), it is necessary to consider the solid temperature along the $x$ direction, i.e. $\frac{d T_{\text {solid }}}{d x}$. Generally 
$T_{\text {solid }}$ is a discontinuous function and needs to be determined from local and global parameters and variables.

Along with the acoustical modeling, it is important to model the piezoelectric alternator in DeltaEC. The flexible diaphragm of the alternator is considered to be an oscillating mass. The DeltaEC model for the alternator removes an amount of acoustic power at the diaphragm equal to the output electrical power produced and small losses in the alternator. Equations describing the alternator are given elsewhere [20].

DeltaEC analysis for a thermoacoustic device is an iterative process. The DeltaEC program needs an initial input file, in which the whole configuration details have to be given as input. For the current configuration, as shown in FIGURE 3, there are 108 parameters that have to be feed into the program, which includes geometrical configuration, boundary conditions, including the RPN (Reverse Polish Notation) calculations for heat transfer, losses due to fluid motion along with the material properties. These inputs are given in the following sections. While designing the thermoacoustic device, the hot heat exchanger plays a major role, since the acoustical power of the system is mainly determined by the amount of energy transferred to the helium working fluid. The following section reports, a stepwise parametric analysis carried out to obtain the optimized configuration for Single Thermal-core Thermoacoustic Electric Generator (ST-TEG).

\section{Parametric study of ST-TEG in DeltaEC}

\section{Effect of exhaust gas pressure drop}

The performance of the thermoacoustic system relies on the careful design of the hot heat exchanger (HHX). The initial iterations of the DeltaEC program for the ST-TEG configuration are carried out to identify the effect of hot exhaust gas pressure drop across the hot heat exchanger. Table 1 shows the assumption of initial and boundary conditions for the study.

\section{Table 1}

\begin{tabular}{|l|l|}
\hline Parameters & Values \\
\hline Working Fluid & Helium \\
\hline Mean Pressure, $\mathrm{p}_{\mathrm{m}}$ & $2.10 \times 10^{6} \mathrm{~Pa}$ \\
\hline Frequency & $300 \mathrm{~Hz}$ \\
\hline Magnitude of pressure oscillation, $\left|\mathrm{p}_{1}\right|$ & $1.65 \times 10^{5} \mathrm{~Pa}$ \\
\hline Phase of pressure oscillation, $\mathrm{Ph}\left(\mathrm{p}_{1}\right)$ & $0 \mathrm{deg}$ \\
\hline $\begin{array}{l}\text { Magnitude of volumetric velocity } \\
\text { oscillation, }\left|\mathrm{U}_{1}\right|\end{array}$ & $0.13 \mathrm{~m}^{3} / \mathrm{s}$ \\
\hline $\begin{array}{l}\text { Phase of volumetric velocity oscillation, } \\
\left.\text { Ph(U }{ }_{1}\right)\end{array}$ & $33 \mathrm{deg}$ \\
\hline $\begin{array}{l}\text { Heat source } \\
\text { Temperature of hot gas }\end{array}$ & $\mathrm{Hot} \mathrm{gas} \mathrm{from} \mathrm{the}$ \\
burner \\
\hline Mass flow rate of hot gas to $\mathrm{HHX}$ & $651 \mathrm{~K}$ \\
\hline
\end{tabular}

In this study of the hot heat exchanger exhaust gas pressure drop, the acoustic network is simplified; the feedback tube and diffusers are not considered. Instead, it is assumed that the acoustic conditions at the top of the ambient heat exchanger are the same as the end of the alternator diaphragm. This implies that the minor losses in the feedback tube and diffusers are ignored in this analysis in order to avoid acoustical complexities.

In the DeltaEC program, the values of the thickness of the hot heat exchanger and the exhaust gas area are allowed to be guessed. The exhaust gas pressure drop is varied and the program is iterated to convergence. Also, the DeltaEC software allows changing the material of the hot heat exchanger. So hot heat exchanger material is varied to stainless steel, Nickel and Molybdenum and the performance is evaluated. The results are plotted as shown in FIGURE 5.

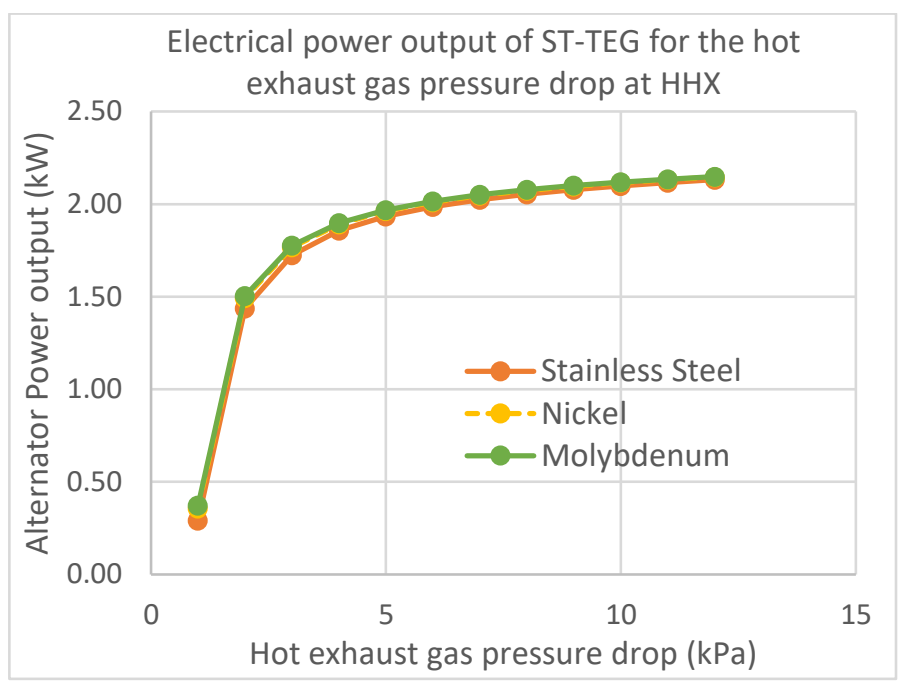

FIGURE 5. PRESSURE DROP OF HOT-GAS AT HHX Vs ALTERNATOR POWER OUTPUT

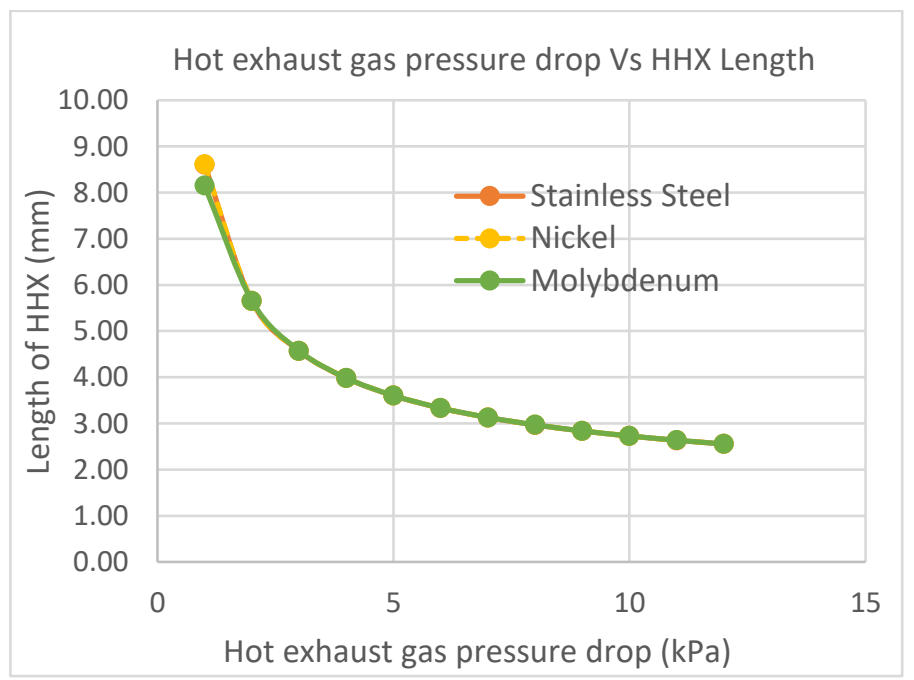

FIGURE 6. PRESSURE DROP OF HOT-GAS AT HHX Vs LENGTH OF HHX 
As the pressure drop of hot exhaust gas across the hot heat exchanger increases, the electrical power output increases. While varying the material, there is no significant variation in alternator power output.

In addition, the thickness of the hot heat exchanger is recorded for every iteration and plotted as shown in FIGURE 6. From this analysis, it is found that the increase in alternator power beyond $4000 \mathrm{~Pa}$ of pressure drop is not significant. Therefore, the values of the pressure drop of $4000 \mathrm{~Pa}$ and the corresponding hot heat exchanger thickness of $4 \mathrm{~cm}$ are taken as the final values for the subsequent parametric study.

\section{Inclusion of feedback tube and diffusers}

In this analysis, the DeltaEC program for the ST-TEG is improved by including the diffusers and feedback tube. The dimensions for those components are finalized by an iterative process. In every iteration, the DeltaEC program is allowed to run several times to achieve the acoustically balanced solution.

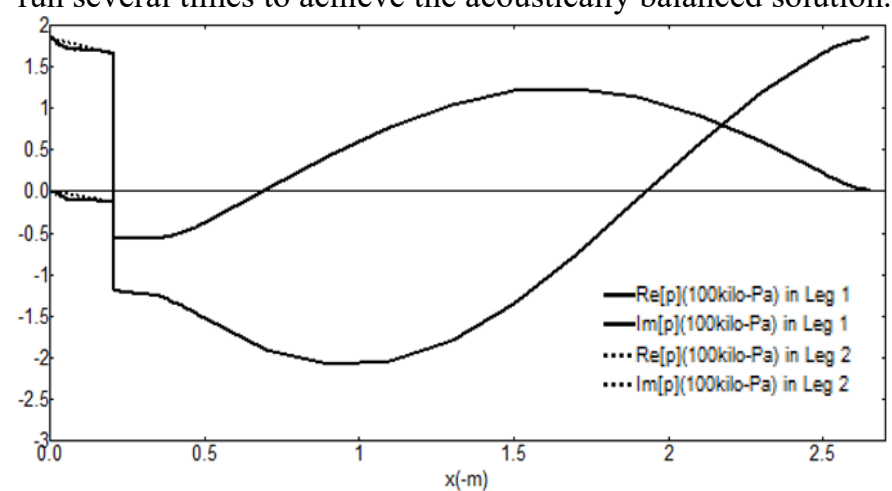

FIGURE 7. REAL AND IMAGINARY PARTS OF ACOUSTIC PRESSURE OBTAINED FROM DELTAEC FOR ST-TEG

FIGURE 7 shows the real and imaginary parts of the acoustic pressure, obtained from the modified DeltaEC program for the ST-TEG configuration. In the plot of FIGURE $7, x$ denotes the coordinate in the direction of wave propagation. This plot shows that the length scale of the feedback tube and diffusers is a little over a half wavelength of the acoustic wave in the device. The length of the feedback tube is taken to be $2 \mathrm{~m}$. The minor and major cross-sectional areas of both diffusers are $3.1 \times$ $10^{-3} \mathrm{~m}^{2}$ and $6.2 \times 10^{-3} \mathrm{~m}^{2}$.

\section{Effect of exhaust gas mass flow rate}

The performance of the thermoacoustic device depends on the amount of thermal energy imparted to the helium gas in the hot heat exchanger. Therefore, the exhaust mass flow rate across the hot heat exchanger becomes the crucial parameter. Moreover, it is important to find the minimum exhaust mass flow rate required to have sound within the thermoacoustic device, known as the onset condition. For this study, conditions are assumed as given in Table 2. The program is modified in such a way that the volumetric velocity oscillation at the beginning of the segment list and the frequency are guessed by DeltaEC. The mass flow rate of hot gas is varied and the results are shown in FIGURE 8.
Table 2

\begin{tabular}{|l|l|}
\hline Parameters & Values \\
\hline Working Fluid & Helium \\
\hline Mean Pressure, $\mathrm{p}_{\mathrm{m}}$ & $2.5 \times 10^{6} \mathrm{~Pa}$ \\
\hline Frequency, $\mathrm{f}$ & $300 \mathrm{~Hz}$ \\
\hline Magnitude of pressure oscillation, $\left|\mathrm{p}_{1}\right|$ & $1.835 \times 10^{5} \mathrm{~Pa}$ \\
\hline Phase of pressure oscillation, $\mathrm{Ph}\left(\mathrm{p}_{1}\right)$ & $0 \mathrm{deg}$ \\
\hline Heat source & $\begin{array}{l}\text { Hot gas from a } \\
\text { burner }\end{array}$ \\
\hline Temperature of hot gas & $623 \mathrm{~K}$ \\
\hline
\end{tabular}

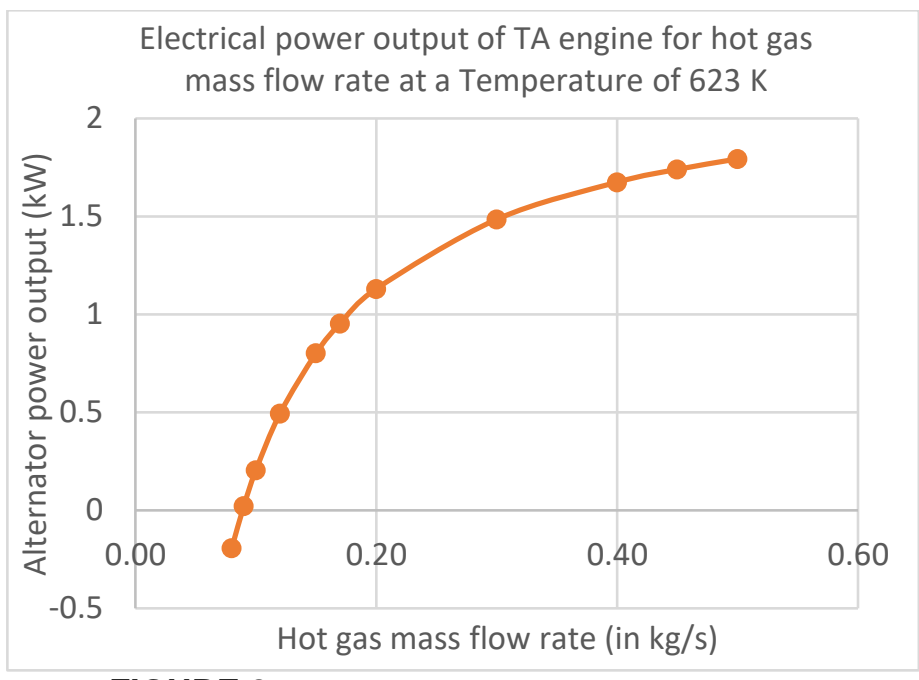

FIGURE 8. HOT GAS MASS FLOW RATE VS ALTERNATOR POWER OUTPUT

When the mass flow rate of hot exhaust gas is $0.5 \mathrm{~kg} / \mathrm{s}$, the obtained electrical power output is $1793 \mathrm{~W}$. From this value the exhaust flow rate is reduced step by step until the power output becomes zero. If the exhaust flow rate is reduced further the simulated alternator power output becomes negative. This is because the DeltaEC program achieves an acoustically balanced condition for the configuration, which requires additional power input. Therefore, it is determined that for the current ST-TEG configuration the lowest flow rate of hot exhaust gas at $623 \mathrm{~K}$ is $0.08 \mathrm{~kg} / \mathrm{s}$ for the onset of sound. Considering the hot exhaust gas mass flow rate and temperature to be at their minimum condition, further analysis is carried out by varying the temperature of the hot gas.

\section{Effect of hot gas temperature on ST-TEG}

The performance of ST-TEG configuration is simulated for a temperature range of $623 \mathrm{~K}$ to $773 \mathrm{~K}$ at two values of the mass flow rate of hot gas; at $0.08 \mathrm{~kg} / \mathrm{s}$ and $623 \mathrm{~K}$, the power output is estimated to be negative. As the temperature increases the electrical output drastically increases. For the case of the hot-gas flow rate of $0.2 \mathrm{~kg} / \mathrm{s}$ and at $623 \mathrm{~K}$, the alternator power output is estimated to be $1130 \mathrm{~W}$, and at $773 \mathrm{~K}$ it is estimated to be 3320 W. Increase in mass flow rate of hot gas source leads to the energy addition to the Helium working fluid across the hot heat exchanger as discussed earlier. FIGURE 9 shows the simulated 
performance of ST-TEG configuration with respect to the temperature variation. As the temperature of the hot heat exchanger increases energy addition to the working fluid increases, which in turn converted as acoustic power. The acoustic power is then converted into electrical power at the alternator.

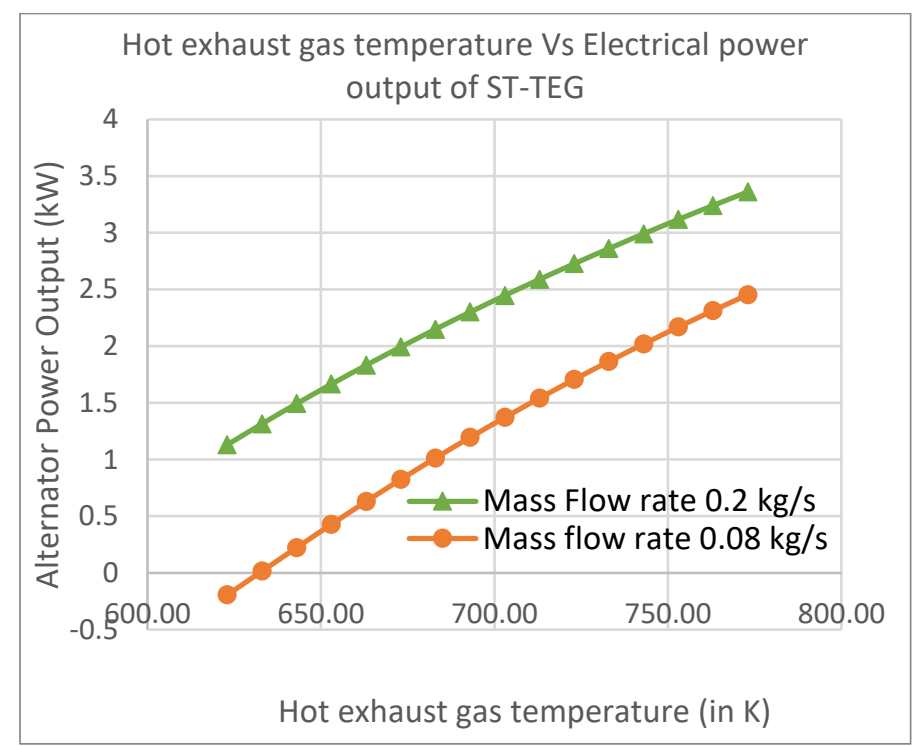

FIGURE 9. HOT EXHAUST GAS TEMPERATURE VS ALTERNATOR POWER

The efficiency of the ST-TEG configuration is evaluated as the alternator power output divided by the thermal power input to the system for the first stage. The temperature drop after the first stage in the observed temperature range is found to be around $40 \mathrm{~K}$. FIGURE 10 shows the efficiency of the thermoacoustic device for a range of hot exhaust gas input temperatures.

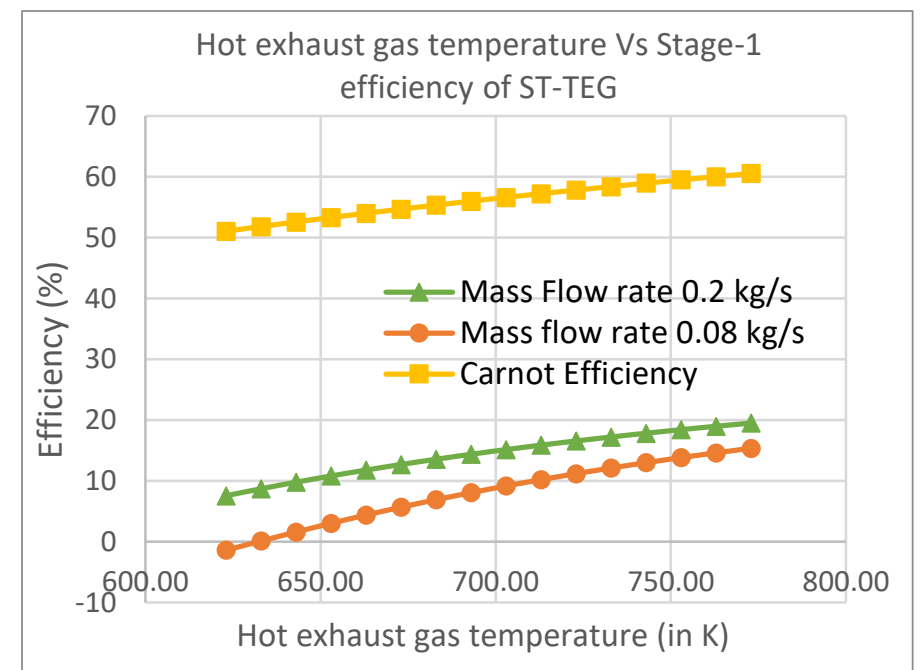

FIGURE 10. HOT EXHAUST GAS TEMPERATURE VS STAGE-1 EFFICIENCY
Also, Carnot efficiency is calculated based on a reference temperature of $305 \mathrm{~K}$ and it is plotted along with the first-stage efficiency of the device. It is evident that the increase in mass flow rate and temperature increases the overall efficiency of the system. Around $20 \%$ of Carnot efficiency is achieved in the first stage.

The device can be designed to have multiple stages of inline. The exhaust hot gas from the first stage can be supplied to subsequent stages. This will fetch more power output and leads to an increase in the overall efficiency of the thermoacoustic device.

\section{CONCLUSION}

The Single Thermal-Core Thermoacoustic Electric Generator (ST-TEG) is intended for producing multi-kilowatts of electrical power output from the hot exhaust of a $20 \mathrm{kWth}$ burner. The device will be the first of its kind thermoacoustic device in generating a kilowatt scale of electricity using a piezoelectric alternator. A parametric study of the device has been carried out using DeltaEC simulation software. The minimum required condition for the hot gas source is estimated from the simulation. The required minimum mass flow rate of hot gas into the hot heat exchanger is $0.08 \mathrm{~kg} / \mathrm{s}$ at a temperature above $623 \mathrm{~K}$. A proper hot heat exchanger has been designed based on the simulation results. The performance of the design configuration is simulated and the results give confidence of producing $3.32 \mathrm{~kW}$ of electrical power at an efficiency of $19.5 \%$ from a hot gas source at a temperature of $773 \mathrm{~K}$ and at an input exhaust flow rate of 0.2 $\mathrm{kg} / \mathrm{s}$.

\section{ACKNOWLEDGMENTS}

We wish to acknowledge the work of Scott Backhaus who wrote the original version of our DeltaEC model.

This study has been supported by the King Abdullah University of Science and Technology through the CARF project 1975-10, and the baseline funds BAS/1/1370-01-01 and BAS/1/1396-01-01.

\section{REFERENCES}

[1] C. Ross, "Overview of Waste Heat Recovery Technologies for Power and Heat," 29 September 2010. [Online]. Available: http://www.northwestchptap.org/NwChpDocs/WHTP\% 20Conference 2010-09-29\%20B.pdf. [Accessed 26 January 2020].

[2] T. Heppenstall, "Advanced gas turbine cycles for power generation: a critical review," Applied Thermal Engineering, vol. 18, no. 9-10, pp. 837-846, 1998.

[3] M. A. G. Timmer, K. D. Block, and T. H. Van der Meer, "Review on the conversion of thermoacoustic power into electricity," The Journal of the Acoustical Society of America, vol. 143, p. 841, 2018. 
[4] G. W. Swift, Thermoacoustics: A unifying perspective for some engines and regenerators, 2nd ed., Springer, 2017.

[5] "LM 6000 PF+ Engine Specifications," Baker Hughes A GE Company, [Online]. Available: https://www.bhge.com/lm6000-pf. [Accessed 20 July 2019].

[6] R. V. L. Hartley, "Electric Power Source". United States of America Patent 2,549,464, 17 April 1951.

[7] A. W. Marrison and B. N. Heights, "Heat-controlled acoustic wave system". United States of America Patent 2836033, 27 May 1958.

[8] P. H. Ceperley, "A pistonless Stirling engine-The traveling wave heat engine," The Journal of the Acoustical Society of America, vol. 66, no. 5, pp. 15081513, 1979.

[9] W. R. Martinin, "Stirling engine design manual," NASA Technical Report, pp. DOE/NASA/3194-1; NASA-CR168088, 1983.

[10] T. Bi, Z. Wu, L. Zhang, G. Yu, E. Luo, and W. Dai, "Development of a $5 \mathrm{~kW}$ traveling-wave thermoacoustic electric generator," Applied Energy, vol. 185, no. 2, pp. 1335-1361, 2017.

[11] K. Wang, S. Dubey, F. H. Choo, and F. Duan, "Thermoacoustic Stirling power generation from LNG cold energy and low-temperature waste heat," Energy, vol. 127, pp. 280-290, 2017.

[12] J. C. Wheately, G. W. Swift and A. Migliori, "Thermoacoustic magnetohydrodynamic electrical generator". The United States Patent 4,599,551, 08 July 1986.

[13] A. Migliori and G. W. Swift, "Liquid-sodium thermoacoustic engine," Applied Physics Letters, vol. 55, no. 5, pp. 355-357, 1988.

[14] K. D. Blok, P. Owczarek and M.-X. Francois, "Bidirectional turbines for converting acoustic wave power into electricity.," in 9th PAMIR international conference on fundamental and applied MHD, Riga, Latvia, 2014.

[15] R. M. Keolian, "Final Report DOE Project DE-FC2604NT42113 Truck thermoacoustic generator and chiller, October 2004-March 2011," 2011.

[16] R. M. Keolian and S. Backhaus, "Energy conversion through thermoacoustics and piezoelectricity," The Journal of the Acoustical Society of America, vol. 130, no. 4, Pt. 2, p. 2504, 2011.

[17] L. Khalij, C. Gautrele, and A. Guillet, "Fatigue curves of a low carbon steel obtained from vibration experiments with an electrodynamic shaker with an electrodynamic shaker," Materials and design, vol. 86, pp. 640-648, 2015.

[18] LANL, "Design Environment for Low-amplitude Thermoacoustic Energy Conversion - DeltaEC Version 6.4b2.7," Los Alamos National Laboratory, 04 December $2017 . \quad$ [Online]. Available: www.lanl.gov/thermoacoustics.

[19] W. C. Ward and G. W. Swift, "Design environment for low-amplitude thermoacoustic engines," The Journal of the Acoustical Society of America, vol. 95, p. 3671, 1994.

[20] R. M. Keolian, J. W. Wuthrich and K. J. Bastyr, "Thermoacoustic piezoelectric generator". United States Patent US 7,772,746, 10 August 2010. 